\title{
Variational approach to the tunneling-time problem
}

\author{
Christian Bracher, Manfred Kleber, and Mustafa Riza \\ Physik-Department T30, Technische Universität München, James-Franck-Straße, D-85747 Garching, Germany
}

(Received 23 February 1999)

\begin{abstract}
Tunneling problems are characterized by different quantum time scales of motion. In this paper, we identify a tunneling time scale, which is based on a simple variational principle. The method utilizes the stationary eigenfunctions for a given one-dimensional potential structure, and it provides a truly local definition of the tunneling time, independent of the asymptotic shape of the potential. We express the minimum tunneling time in terms of the more common time scales obtained from the Larmor clock setup. Asymptotic formulas for both the extreme quantum and the semiclassical limit are presented. As an experimental verification of the variational approach we demonstrate that the minimum tunneling time governs the time a particle requires to traverse the barrier in a symmetric double-well structure. [S1050-2947(99)04209-2]
\end{abstract}

PACS number(s): 03.65.-w, 73.40.Gk

\section{INTRODUCTION}

In recent years, one of the most controversial debates regarding the foundations of physics dealt with the time spent by a quantum particle traversing a given sector in space. Even though the problem is not limited to potential barrier penetration, the issue became notorious as the "tunneling time problem." Despite decades of discussion, no unanimously accepted solution emerged; rather, differing proposals for the quantum-mechanical sojourn time abound [1-3]. Lately, experiments indicating superluminal transmission of photons through "tunneling barriers" built of mismatched wave guides [3-5] and multilayer mirrors [6] stirred renewed interest in the problem.

Thus, we are faced with the curious situation that to a seemingly definite and simple question, various answers prevail that are not necessarily compatible with each other. It seems that much of the trouble in defining the tunneling time is rooted in our conception of a "clock." The notion of an external stopwatch timing some process without influencing the event is an intrinsically classical idea, and one should not be surprised that this classical picture breaks down in the quantum limit. In fact, there is no unifying "clock principle" in the quantum realm, but every attempt to identify the evolution of some physical observable with the elapsed time leads to its own proprietary set of quantum time scales of motion. Evidently, the readings of these quantum clocks are supposed to match in the classical limit. However, there is no way to reverse this process and select a unique "proper", quantum clock. Rather, the tunneling time depends on how one sets out to measure it.

Our subjective assessment of the situation delivers a mixed message. On the one hand, it obviously implies that the quest for a definite tunneling time is doomed to failure. Yet, it also opens the way for further alternative definitions of the tunneling time. In order to be useful, rather than being mere theoretical constructs these newly proposed quantities should be based on a physical property of the tunneling process under consideration.

The possibility of tunneling is almost inevitably connected with the process of reflection. This wave-mechanical feature entangles the tunneling-time problem with the probability and the duration of particle reflection from a potential structure. For example, in the case of symmetric potential barriers, the Wigner phase time for tunneling [7] and for reflection are identical quantities. The same property is valid for the corresponding phase times obtained from the Larmor clock approach [8-10]. Such a result is not easily interpreted, in particular for extended barriers, where reflection dominates and particles apparently are accelerated in tunneling (Hartman effect). However, there is one situation where tunneling without reflection occurs [11]: In resonant tunneling through the central barrier of a symmetric double-well potential, the quantum particle may oscillate between the two wells. This problem is unique in the sense that this transmission process periodically takes place with unit probability, while reflection is absent.

In our contribution, we elucidate how the switching time, which is observed in an experiment as a splitting of degenerate energy levels in the symmetric double well, is connected to a general expression $\tau_{\min }(E)$ for the tunneling time that we will denote the minimum tunneling time as it yields a time scale for stationary tunneling processes founded upon a simple variational principle. Having introduced the time scale, which is related in concept to the dwell time $\tau_{\mathrm{S}}(E)$ originally devised by Smith [12], we proceed to derive explicit expressions for $\tau_{\text {min }}(E)$, including a representation in terms of the set of Larmor clock times, the workhorse for comparisons between different quantum clocks. Subsequently, we inquire into the properties of the minimum tunneling time, in particular, its asymptotic behavior. Finally, the results of the theory are illustrated by means of some simple examples.

\section{COMMON APPROACHES}

It is instructive to first present a brief overview of some common definitions for the tunneling time, which we will use to motivate our proposal of $\tau_{\min }(E)$. We also state the results of the Larmor clock model in a compact fashion [8-10] for reference purposes. (As indicated above, nothing is implied by not mentioning some of the major approaches to the tunneling-time problem in this section.)

\section{A. The Bohmian dwell time}

Here, we are concerned with a nonrelativistic description of one-dimensional stationary quantum motion. Hence, our 


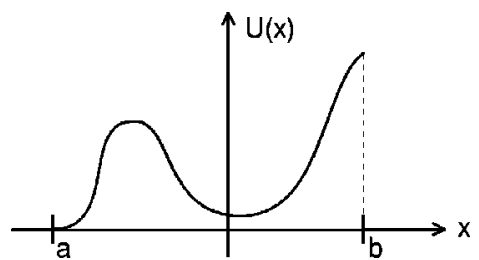

FIG. 1. A finite-range potential barrier $U(x)$ extending from $x=a$ to $x=b$.

object of interest is the continuous, doubly degenerate spectrum of eigenstates $\Psi_{E}(x)$ of the stationary Schrödinger equation (for the sake of simplicity, let us omit any vector potential):

$$
\left[-\frac{\hbar^{2}}{2 m} \frac{\partial^{2}}{\partial x^{2}}+U(x)\right] \Psi_{E}(x)=E \Psi_{E}(x) .
$$

To each solution $\Psi_{E}(x)$, we assign as a functional the (spatially constant) probability current $j\left[\Psi_{E}\right]$ defined as usual by

$$
j\left[\Psi_{E}\right]=\frac{\hbar}{m} \operatorname{Im}\left[\Psi_{E}^{*}(x) \frac{\partial}{\partial x} \Psi_{E}(x)\right] .
$$

Now, a conceptually simple definition for the time spent by a quantum particle in the interval $a<x<b$ may be given by the following scheme:

$$
\tau_{\mathrm{D}}\left[\Psi_{E}\right]=\frac{1}{\left|j\left[\Psi_{E}\right]\right|} \int_{a}^{b} d x\left|\Psi_{E}(x)\right|^{2} .
$$

Formally, $\tau_{\mathrm{D}}\left[\Psi_{E}\right]$ denotes the time required by the current $j\left[\Psi_{E}\right][$ Eq. (2)] to replace the particles present in the interval (the barrier) $a<x<b$. This scaling invariant expression is therefore inspired by a hydrodynamical model of quantum mechanics, so it should not come as a surprise that in the framework of the Bohm interpretation of quantum mechanics, the tunneling time takes on form (3) [13].

\section{B. Smith's dwell time}

The original approach by Smith [12] subtly differs from the above development. Smith was interested in isolated potential barriers, which are limited to the range $a<x<b$; otherwise, $U(x)$ should vanish (see Fig. 1). In this situation, those special solutions $\Psi_{E}^{\overrightarrow{ }}(x), \Psi_{E}^{\leftarrow}(x)$ of Eq. (1), which behave as outgoing waves for $x \rightarrow \pm \infty$, play a prominent role. Clearly, outside the barrier these eigenstates are entirely fixed by the reflection and transmission amplitudes of the potential $U(x)\left(k^{2}=2 m E / \hbar^{2}\right)$ :

$$
\begin{aligned}
& \Psi_{E}^{\vec{E}}(x) \propto \begin{cases}e^{i k x}+|R(E)| e^{i \rho(E)} e^{-i k x} & (x<a) \\
|T(E)| e^{i \delta(E)} e^{i k x} & (x>b),\end{cases} \\
& \Psi_{E}^{\leftarrow}(x) \propto \begin{cases}|T(E)| e^{i \delta(E)} e^{-i k x} & (x<a) \\
e^{-i k x}+|R(E)| e^{i \sigma(E)} e^{i k x} & (x>b) .\end{cases}
\end{aligned}
$$

In passing we note that the complex reflection and transmission amplitudes $R(E)$ and $T(E)$ are not completely independent, but subject to restrictions imposed by unitarity requirements. These not only enforce the equality of the left-hand and right-hand transmission amplitudes $T(E)$ in Eqs. (4) and (5), but also interrelate reflection and transmission quantities [10]:

$$
\begin{gathered}
|R(E)|^{2}+|T(E)|^{2}=1, \\
\rho(E)+\sigma(E)=\pi+2 \delta(E) .
\end{gathered}
$$

Later on, we will connect these relations to the readings of the Larmor clock.

In the asymptotic sectors $x<a$ and $x>b$ we may interpret the total current $j\left[\Psi_{E}\right]$ for eigenstates (4) and (5) as the difference of an incoming current $j_{\text {inc }}$ and a reflected current $j_{\text {refl }}$. In Smith's original definition of the dwell time, in Eq. (3) the total current $j\left[\Psi_{E}\right]$ is replaced by the incoming current $j_{\text {inc }}$. Consequently, Smith's dwell times $\tau_{\mathrm{S}}(E)$ and $\tau_{\mathrm{S}}^{\leftarrow}(E)$ (which generally differ for nonsymmetric barriers) are linked to our definition of $\tau_{\mathrm{D}}\left[\Psi_{E}\right]$ via the transmission probability $|T(E)|^{2}$ of the barrier:

$$
\tau_{\mathrm{S}}(E)=\frac{1}{j_{\text {inc }}} \int_{a}^{b} d x\left|\Psi_{E}(x)\right|^{2}=|T(E)|^{2} \tau_{\mathrm{D}}\left[\Psi_{E}^{\vec{E}}\right]
$$

[An analogous relation holds for $\tau_{\mathrm{S}}^{\leftarrow}(E)$.]

Obviously, unlike prescription (3), Smith's original definition of the dwell time $\tau_{\mathrm{S}}(E)$ works only for finite-range potential barriers and outgoing waves, i.e., it implicitly depends on the asymptotic behavior of $\Psi_{E}(x)$. Hence, it is not a local time scale in the sense that knowledge of $U(x)$ in the range $a<x<b$ suffices to determine the tunneling time, a criterion that is met by the definition of $\tau_{D}\left[\Psi_{E}\right]$ in Eq. (3). Thus, we will continue to work with the latter expression, which we nevertheless refer to as dwell time in the following.

\section{The Larmor clock}

One of the most fruitful approaches to the tunneling-time problem is the Larmor clock model first put forward by Baz' [14]. Here, we employ the notation used in refined treatments of this gedanken experiment [8-10]. Optical analogues of these time scales have been accessed in experiments using frustrated internal reflection of light $[15,16]$.

Formally, we obtain the Larmor time scales through the following setup. Consider the finite-range potential $U(x)$ depicted in Fig. 1. We now perturb the barrier potential by superimposing an infinitesimal variational step potential $V(x)$ covering the barrier:

$$
V(x)=V \Theta(x-a) \Theta(b-x) .
$$

Then, the Larmor times are defined as the logarithmic derivatives of the reflection and transmission amplitudes $R(E, V)$ and $T(E, V)$ of the barrier with respect to the perturbation strength $V$ at $V=0$. It is somewhat surprising to see that this linear-response theory leads to a set of two complex, or, respectively, four real tunneling time scales: 


$$
\begin{aligned}
\tau_{R}^{y, \rightarrow}(E)+i \tau_{R}^{z}(E) & =\left.\frac{i \hbar}{R^{\rightarrow}(E)} \frac{\partial R^{\rightarrow}(E, V)}{\partial V}\right|_{V=0} \\
& =-\hbar \frac{\partial \rho(E)}{\partial V}+i \hbar \frac{\partial \ln |R(E)|}{\partial V} \\
\tau_{T}^{y}(E)+i \tau_{T}^{z}(E) & =\left.\frac{i \hbar}{T(E)} \frac{\partial T(E, V)}{\partial V}\right|_{V=0} \\
& =-\hbar \frac{\partial \delta(E)}{\partial V}+i \hbar \frac{\partial \ln |T(E)|}{\partial V}
\end{aligned}
$$

Equivalent definitions hold for waves $\Psi_{E}^{\leftarrow}(x)$ [Eq. (5)] impinging on the right-hand side of the barrier. Unitarity restrictions (6) and (7) imposed on their parent amplitudes imply corresponding sum rules for the Larmor time scales:

$$
\begin{gathered}
|R(E)|^{2} \tau_{R}^{z}(E)+|T(E)|^{2} \tau_{T}^{z}(E)=0, \\
\tau_{R}^{y, \rightarrow}(E)+\tau_{R}^{y, \leftarrow}(E)=2 \tau_{T}^{y}(E) .
\end{gathered}
$$

Therefore, only three independent Larmor time scales exist. We also note that for symmetric potential barriers $U(x)$ $=U(-x)$, Eq. (13) implies that the Larmor clock readings for reflected and transmitted waves coincide: $\tau_{R}^{y, \rightarrow}(E)$ $=\tau_{R}^{y, \leftarrow}(E)=\tau_{T}^{y}(E)$.

Although it appears difficult to assign an unambiguous physical interpretation to individual Larmor times, their versatility renders them a powerful tool in the analysis of the tunneling-time problem. Most proposals for the tunneling time may be restated as various combinations of the Larmor time scales $\tau_{R}^{y}(E), \tau_{T}^{y}(E), \tau_{R}^{z}(E)$, and $\tau_{T}^{z}(E)$, which qualifies them for comparative studies of quantum clocks. For example, Smith's dwell time $\tau_{\mathrm{S}}(E)$ (8) adopts the form of a weighed " $y$ " Larmor time average:

$$
\tau_{\mathrm{S}}(E)=|R(E)|^{2} \tau_{R}^{y, \rightarrow}(E)+|T(E)|^{2} \tau_{T}^{y}(E) .
$$

(In the case of symmetric potential barriers, $\tau_{\mathrm{S}}(E)$ and $\tau_{T}^{y}(E)$ are also identical.) In a similar vein, the minimum tunneling time $\tau_{\min }(E)$, which we are about to define, is amenable to a description in terms of the Larmor times.

\section{A MINIMUM TUNNELING TIME}

Keeping these preliminary remarks in mind, we now proceed to define a variationally determined tunneling time scale $\tau_{\min }(E)$. We start out our discussion from the Bohmian result for the dwell time $\tau_{D}\left[\Psi_{E}\right]$ [Eq. (3)] that we motivated in Sec. II A. There, we noted that this particular time scale is a functional of the eigenstate $\Psi_{E}(x)$ of Schrödinger equation (1). However, it appears preferable to deal with a tunneling time that, apart from the particle energy $E$, depends solely on the potential relief $U(x)$ in the interval of interest $a<x<b$ [17]. (After all, this property holds true for classical transmission over the barrier.) In order to transform the functional $\tau_{D}\left[\Psi_{E}\right]$ into a time scale independent of our choice of wave function $\Psi_{E}(x)$ in Eq. (3), we employ a simple variational principle: Clearly, $\tau_{D}\left[\Psi_{E}\right]$ is a positively definite quantity, which must be bounded from below; it continuously varies in the two-dimensional state space of eigenfunctions $\Psi_{E}(x)$ in the interval $a<x<b$. Hence, for some special solutions $\Psi_{\text {min }}^{E}(x)$ of Eq. (1) the dwell time $\tau_{D}\left[\Psi_{E}\right]$ assumes its minimum value, which we shall denote as the minimum tunneling time $\tau_{\min }(E)$ in the potential structure $U(x)$ for the interval $a<x<b$ :

$$
\tau_{\min }(E)=\min _{H \Psi=E \Psi}\left[\frac{1}{|j[\Psi]|} \int_{a}^{b} d x|\Psi(x)|^{2}\right] .
$$

Correspondingly, we call any eigenstate $\Psi_{\min }^{E}(x)$ of Eq. (1) that minimizes Eq. (15) a minimal wave function of the potential barrier $U(x)$ in the interval $a<x<b$. We should point out that no maximum value of the dwell time functional $\tau_{D}\left[\Psi_{E}\right]$ exists as it diverges for solutions $\Psi_{E}(x)$ that do not carry any current, $j\left[\Psi_{E}\right]=0$. This happens, e.g., for real solutions of Eq. (1). For the sake of clarity, we remark that the term "minimal tunneling time" refers to the origin of this time scale in a variational procedure. We do not claim that $\tau_{\min }(E)$ presents a universal lower bound for tunnelingtime proposals that are based upon a different principle.

As an immediate consequence of its definition (15), we note that the minimum tunneling time $\tau_{\min }(E)$ does not present an additive quantity. Assume that the interval $a<x$ $<b$ is split into two subintervals $a<x<c$ and $c<x<b$. Then, from the variation in Eq. (15) the inequality readily follows:

$$
\tau_{\min }(a, b ; E) \geqslant \tau_{\min }(a, c ; E)+\tau_{\min }(c, b ; E) .
$$

Obviously, equality in Eq. (16) should occur in the limit of classical motion $[E \gg U(x)]$. For particle tunneling, i.e., $E$ $\ll U(x)$, the additivity property does not nearly hold. (See also, Sec. V B.)

Unlike most other candidates for the tunneling time, our contender $\tau_{\min }(E)$ shows the advantage of being a locally determined quantity. Let us elaborate this notion: The common definitions of Smith's dwell time $\tau_{\mathrm{S}}(E)$ [Eq. (8)] and the Larmor times $\tau_{R}^{y, \rightarrow}(E), \tau_{T}^{y}(E), \tau_{R}^{z}(E)$, and $\tau_{T}^{z}(E)$ [Eqs. (10) and (11)] are all founded upon outgoing-wave solutions and thus implicitly depend on imposed boundary conditions. Any change of the potential $U(x)$ outside the range $a<x$ $<b$ will cause a different selection of outgoing wave states; as a consequence, Smith's dwell time and the Larmor times for quantum motion in the interval $a<x<b$ will be affected. Hence, these time scales depend on global properties of $U(x)$. In contrast, by definition (15) the minimum tunneling time $\tau_{\min }(E)$ is wholly determined by the set of eigenfunctions $\Psi_{E}(x)$ in the examined interval $a<x<b$. But this set is completely fixed by the choice of energy $E$ and the topography of the potential $U(x)$ in this interval. Thus, both $\tau_{\min }(E)$ and the corresponding set of minimal wave functions $\Psi_{\min }^{E}(x)$ are truly local quantities. This property makes them applicable in situations where no outgoing wave solutions exist; we present an example in Sec. VI.

Finally, we remark that the minimal eigenstates $\Psi_{\min }^{E}(x)$ are given, apart from physically irrelevant scalings $\Psi$ $\rightarrow \lambda \Psi$, by a unique pair of complex conjugate wave functions. [Note that in quantum mechanics, complex conjuga- 
tion of the wave function corresponds to a time reversal operation, which merely changes the sign of the current $j\left[\Psi_{E}\right]$ in Eq. (2).]

\section{EXPLICIT EXPRESSIONS}

From the variational principle stated in Eq. (15), we now derive an explicit representation for the minimum tunneling time $\tau_{\min }(E)$ and the corresponding wave functions $\Psi_{\min }^{E}(x)$ in terms of an arbitrary basis of eigenfunctions in the barrier region $a<x<b$. For a finite-range barrier, we also may express $\tau_{\min }(E)$ in terms of the common Larmor time scales (Sec. II C).

\section{A. Representation by eigenfunctions}

First, we show how to obtain $\tau_{\min }(E)$ and $\Psi_{\text {min }}^{E}(x)$ from a pair of linearly independent real eigenstates $c(x)$ and $s(x)$ of Hamiltonian (1) with energy $E$. Our treatment, which is very general, only requires that the Wronskian determinant of these solutions be normalized:

$$
\mathcal{W}[s, c]=s(x)^{\prime} c(x)-s(x) c(x)^{\prime}=1 .
$$

This property may always be achieved by a simple scaling operation on $s(x)$. (Note that this normalization procedure, if performed symmetrically on $c(x)$ and $s(x)$, leads to dimensional units [length $]^{1 / 2}$ for these wave functions. Although unusual, this normalization scheme has the advantage that $c(x)$ and $s(x)$ do not depend on the interval boundaries $a$ and $b$.)

To find the minimum tunneling time and minimum wave functions, we form a general linear combination of the basis functions $\Psi_{E}(x)=\alpha c(x)+\beta s(x)$, introduce it into the defining equation of dwell time [Eq. (3)], and perform the variation of the complex parameters $\alpha, \beta$ [Eq. (15)]. This straightforward procedure leads to simple and elegant expressions for the requested quantities. For $\tau_{\min }(E)$, we find

$$
\begin{aligned}
\tau_{\min }(E)= & \frac{2 m}{\hbar}\left\{\int_{a}^{b} d x c(x)^{2} \int_{a}^{b} d x s(x)^{2}\right. \\
& \left.-\left[\int_{a}^{b} d x s(x) c(x)\right]\right\}^{1 / 2} .
\end{aligned}
$$

Note that the Cauchy-Schwarz inequality for integrals [18] guarantees a positive definite radicand in this expression, which is furthermore independent of the actually employed set of basis functions $c(x)$ and $s(x)$, provided that condition (17) is satisfied. A possible choice for a pair of conjugate complex minimal wave functions $\Psi_{\min }^{E}(x)$ is given by the linear combination,

$$
\Psi_{\min }^{E}(x) \propto c(x)-\left(\frac{\int_{a}^{b} d \xi c(\xi)^{2}}{\int_{a}^{b} d \xi s(\xi)^{2}}\right)^{1 / 2} \exp \left\{ \pm i \arccos \frac{\int_{a}^{b} d \xi s(\xi) c(\xi)}{\sqrt{\int_{a}^{b} d \xi c(\xi)^{2} \int_{a}^{b} d \xi s(\xi)^{2}}}\right\} s(x) .
$$

[We remark that this formula contains as phase the abstract angle between the eigenstates $c(x)$ and $s(x)$ in the normed space $L^{2}(a, b)$ of square-integrable functions.] For the special case of symmetric barriers $[U(x)=U(-x), a=-b]$, expressions (18) and (19) may be considerably simplified. Exploiting the symmetry properties of the potential, we may select even and odd-parity eigenstates as basis functions $c(x)=c(-x)$ and $s(x)=-s(-x)$ in these formulas. This procedure yields

$$
\begin{gathered}
\tau_{\min }(E)=\frac{4 m}{\hbar} \sqrt{\int_{0}^{b} d x c(x)^{2} \int_{0}^{b} d x s(x)^{2}} \\
\Psi_{\min }^{E}(x) \propto \sqrt{\int_{0}^{b} d \xi s(\xi)^{2} c}(x) \pm i \sqrt{\int_{0}^{b} d \xi c(\xi)^{2}} s(x) .
\end{gathered}
$$

\section{B. Connection to the Larmor clock}

If we restrict ourselves to potential barriers $U(x)$ that are confined to the interval $a<x<b$ (Fig. 1), we may use the set of outgoing waves $\Psi_{E}(x), \Psi_{E}^{\leftarrow}(x)$ [Eqs. (4) and (5)] as a basis in the state space of eigenfunctions $\Psi_{E}(x)$. Note that the integral appearing in the definition of the dwell time
$\tau_{\mathrm{D}}\left[\Psi_{E}\right][$ Eq. (3)] may be expressed in terms of Larmor times (10) and (11). Hence, by variation of the wave function, we are able to represent the minimum tunneling time $\tau_{\min }(E)$ in terms of the transmission probability of the barrier and its set of Larmor clock time scales. A tedious calculation finally leads to a fairly compact expression:

$$
\begin{aligned}
\tau_{\min }(E)= & \frac{1}{|T(E)|}\left\{|R(E)|^{2} \tau_{R}^{y, \rightarrow}(E) \tau_{R}^{y, \leftarrow}(E)\right. \\
& \left.+|T(E)|^{2} \tau_{T}^{y}(E)^{2}+\tau_{R}^{z}(E) \tau_{T}^{z}(E)\right\}^{1 / 2} .
\end{aligned}
$$

Note that the whole set of left-hand and right-hand Larmor time scales enters this functional. In the same manner, the minimal wave function $\Psi_{\min }^{E}(x)$ might be represented in terms of the Larmor time scales and the outgoing waves $\Psi_{E}^{\overrightarrow{ }}(x)$ and $\Psi_{E}^{\leftarrow}(x)$. The relevant expression is, however, extraordinarily complicated, and we refrain from displaying it here.

\section{ASYMPTOTIC BEHAVIOR}

Having explicit representation (18) for the minimum tunneling time $\tau_{\min }(E)[E q .(15)]$ at hand, we now proceed to 
establish the behavior of this tunneling time scale in the limiting cases of extreme quantum dynamics and semiclassical motion. To define these regimes, we may employ the modulus of the classical action functional $S_{\mathrm{cl}}(a, b ; E)$ as a characteristic quantity of the barrier potential $U(x)$ :

$$
S_{\mathrm{cl}}(a, b ; E)=\int_{a}^{b} d x \sqrt{2 m[E-U(x)]} .
$$

If $\left|S_{\mathrm{cl}}(a, b ; E)\right| \ll \hbar$, the wave function $\Psi_{E}(x)$ is hardly affected by the barrier. In the opposite regime $\left|S_{\mathrm{cl}}(a, b ; E)\right|$ $\gg \hbar$, the problem is usually amenable to a treatment in the framework of the WKB approximation.

\section{A. The narrow barrier limit}

Let us first study ultrathin barriers with $\left|S_{\mathrm{cl}}(a, b ; E)\right| \ll \hbar$. In this case, the radius of curvature of the wave function $\Psi_{E}(x)$ in the barrier is large compared to the extension $d$ $=b-a$ of the interval of interest, so we may neglect quadratic and higher terms in $d$ in the Taylor expansion of $\Psi_{E}(x)$ with respect to $x=a$. Under these circumstances, for the calculation of $\tau_{\min }(E)$ we may always employ the pair of approximate eigenfunctions $c(x)=1$ and $s(x)=x-a$ as a normalized function basis in Eq. (18). This linear approximation immediately yields

$$
\tau_{\min }(E)=\frac{1}{\sqrt{3}} \frac{m d^{2}}{\hbar}+\mathcal{O}\left(d^{4}\right)
$$

In the narrow barrier limit, the maximum transmission velocity $\mathrm{v}_{\min }=d / \tau_{\min }(E)$ is, therefore, given by the universal expression $\mathrm{v}_{\min }=\sqrt{3} \hbar / m d$. Note that $\mathrm{v}_{\text {min }}$ depends, apart from natural constants, only on the barrier width $d$, but is independent of the energy $E$. This property may be viewed as a consequence of the Heisenberg uncertainty relation; indeed, we may restate Eq. (24) in the alternative form $\left(m \mathrm{v}_{\min }\right) d$ $\approx \sqrt{3} \hbar$. [As $d \rightarrow 0, \mathrm{v}_{\text {min }}$ may assume arbitrarily large values. This unphysical behavior could be corrected by starting with the proper relativistic wave equation instead of Schrödinger equation (1).]

\section{B. The semiclassical limit}

For $\left|S_{\mathrm{cl}}(a, b ; E)\right| \gg \hbar$, application of a semiclassical theory is in order. We may distinguish between two entirely different regimes, viz., quasiclassical transmission over the barrier for $E \gg U(x)$, and the case of barrier penetration where $E$ $\ll U(x)$.

For convenience, we introduce the action functional $S(x ; E)=\left|S_{\mathrm{cl}}(a, x ; E)\right|$ and the abbreviation $\Sigma(x ; E)$ :

$$
\begin{gathered}
S(x ; E)=\left|\int_{a}^{x} d \xi \sqrt{2 m[E-U(\xi)]}\right|, \\
\Sigma(x ; E)=\left[\frac{\hbar^{2}}{2 m|E-U(x)|}\right]^{1 / 4} .
\end{gathered}
$$

Let us now first inquire into the quasiclassical limit $E$ $\gg U(x)$. Then, a pair of WKB solutions $c_{\mathrm{WKB}}(x)$ and $s_{\mathrm{WKB}}(x)$ is given by

$$
\begin{aligned}
& c_{\mathrm{WKB}}(x)=\Sigma(x ; E) \cos [S(x ; E) / \hbar], \\
& s_{\mathrm{WKB}}(x)=\Sigma(x ; E) \sin [S(x ; E) / \hbar] .
\end{aligned}
$$

Note that even though these functions are only approximate solutions to the Schrödinger equation, they show a spatially constant Wronskian determinant which is already normalized in the sense of Eq. (17). Thus we may insert the pair $c_{\mathrm{WKB}}(x)$ and $s_{\mathrm{WKB}}(x)$ into Eq. (18) to obtain the WKB approximation to the minimum tunneling time in the limit of quasiclassical transmission. After a few transformations, we find

$$
\tau_{\min }^{\mathrm{WKB}}(E)=\sqrt{\tau_{\mathrm{cl}}(E)^{2}-\left\{\int_{a}^{b} \frac{d x}{\mathrm{v}_{\mathrm{cl}}(x ; E)} \cos \left[\frac{2}{\hbar} S(x ; E)\right]\right\}^{2}-\left\{\int_{a}^{b} \frac{d x}{\mathrm{v}_{\mathrm{cl}}(x ; E)} \sin \left[\frac{2}{\hbar} S(x ; E)\right]\right\}^{2}} .
$$

Here, $\tau_{\mathrm{cl}}(E)$ denotes the classical time of flight from $x=a$ to $x=b$, whereas $\mathrm{v}_{\mathrm{cl}}(x ; E)$ represents the classical particle velocity in the potential $U(x)$. From Eq. (29) we infer that $\tau_{\min }(E)$ indeed presents a valid quantum clock since it coincides with the classical transmission time $\tau_{\mathrm{cl}}(E)$ in the limit $\hbar \rightarrow 0$. As expected, $\tau_{\min }^{\mathrm{WKB}}(E) \leqslant \tau_{\mathrm{cl}}(E)$ holds.

Let us finally examine the tunneling case $E \ll U(x)$. In this case, we have to replace oscillating functions (27) and (28) by evanescent waves:

$$
\begin{gathered}
c_{\mathrm{WKB}}(x)=\frac{1}{2} \Sigma(x ; E) \exp [-S(x ; E) / \hbar], \\
s_{\mathrm{WKB}}(x)=\Sigma(x ; E) \exp [S(x ; E) / \hbar] .
\end{gathered}
$$

Using this normalized pair of basis functions, from Eq. (18) we arrive after some reorderings at the following WKB approximation for the minimum tunneling time in the thick barrier penetration limit:

$$
\tau_{\min }^{\mathrm{WKB}}(E)=\frac{\hbar}{4 \sqrt{[U(b)-E][U(a)-E]}} \exp \left[\frac{1}{\hbar}\left|S_{\mathrm{cl}}(a, b ; E)\right|\right] .
$$

In the case of tunneling, $\tau_{\min }(E)$ rises exponentially with the width of the barrier $d$. The minimum tunneling time does not show the accelerating effect of tunneling gaps predicted by several other theories of the tunneling time and observed in evanescent photon modes $[4,6]$. We note that the minimum 


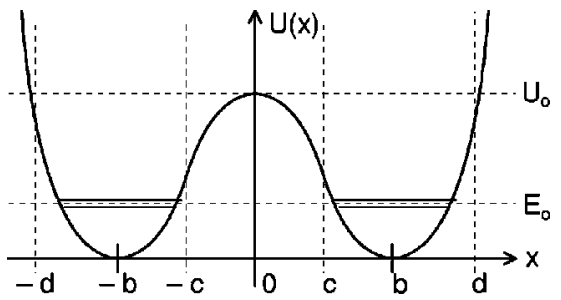

FIG. 2. A symmetric double-well potential structure built of parabolic potentials.

tunneling time is roughly inversely proportional to the modulus of the barrier transmission amplitude $|T(E)|$.

\section{APPLICATION TO DOUBLE-WELL STRUCTURES}

So far, our considerations regarding the minimum tunneling time $\tau_{\min }(E)$ have been of an entirely mathematical nature. Yet the preponderance of theoretical reflections over practical applications has always been a troublesome aspect of the discussion about the tunneling-time problem. Therefore, it appears important to identify physical processes that permit us to extract the quantum time scales of motion in an actual experiment rather than to content oneself with a "gedankenversuch." The time scale $\tau_{\min }(E)$ quite naturally emerges in the quantum mechanics of double-well potentials as is elaborated below.

\section{A. Level splitting}

Let us examine the following setup (Fig. 2): The symmetric potential $U(x)$ is composed of two wells centered around $x= \pm b$, which are separated by a central potential barrier. As $x \rightarrow \pm \infty$, outer barriers isolate the double-well structure from the environment. Each well gives rise to a discrete spectrum of eigenstates with energies $E_{n}$, but since the structure is assumed symmetric, the states in the left and right wells are in resonance, and the double degeneracy of the eigenstates will be lifted by the possibility of electron tunneling through the central barrier. This causes a small shift of the eigenenergies $E_{n}$, which will split into close doublets $\widetilde{E}_{n} \pm \Delta E_{n} / 2$ separated by the level splitting $\Delta E_{n}$. Because these doublets are composed of states of opposite parity, transitions between them may be enforced by electromagnetic dipole radiation, which allows for a precise determination of the level splitting. As a typical example, we refer to the fine structure of the states of the ammonia molecule caused by oscillation, which may be observed as maser radiation. A simple presentation is given in Feynman's textbook [19].

Let us become more specific. Assume that $H_{\text {well }}$ denotes the Hamiltonian of a single well, say, the right one. Then, the well eigenstates are given by

$$
H_{\text {well }} \Psi_{n}(x)=E_{n} \Psi_{n}(x) .
$$

Due to the presence of the other well, these eigenstates split into states of definite parity $\Psi_{n}^{(e)}(x)$ and $\Psi_{n}^{(o)}(x)$ of the complete system:

$$
\begin{aligned}
& \Psi_{n}^{(e)}(x) \approx \alpha\left[\Psi_{n}(x)+\Psi_{n}(-x)\right], \\
& \Psi_{n}^{(o)}(x) \approx \beta\left[\Psi_{n}(x)-\Psi_{n}(-x)\right] .
\end{aligned}
$$

Here, we fix the prefactors $\alpha$ and $\beta$ by the requirement that at the center of symmetry $x=0, \Psi_{n}^{(e)}(0)=1$ and $\Psi_{n}^{(o)}(0)^{\prime}$ $=1$ holds. With this choice, the Wronskian determinant of both functions will be normalized at $x=0$, which obviously eases the connection to the developments of Sec. IV. States (34) and (35) are eigenstates of the complete Hamiltonian $H$ :

$$
\begin{aligned}
& H \Psi_{n}^{(e)}(x)=\left(\widetilde{E}_{n}-\frac{\Delta E_{n}}{2}\right) \Psi_{n}^{(e)}(x), \\
& H \Psi_{n}^{(o)}(x)=\left(\widetilde{E}_{n}+\frac{\Delta E_{n}}{2}\right) \Psi_{n}^{(o)}(x) .
\end{aligned}
$$

The deformation of the well potential leads to a deviation from the original eigenenergy $\widetilde{E}_{n}-E_{n}$ that is, however, exponentially small in the WKB sense. The same statement holds for the splitting $\Delta E_{n}$ caused by tunneling.

We now have to look for the connection with the tunneling time through the central barrier. To this end, we examine the evolution of a state that starts out in the right potential well. From Eqs. (34) and (35) we infer that such a state for $t=0$ is initially given by $\Psi_{E}^{(r)}(x, 0)=\beta \Psi_{n}^{(e)}(x)+\alpha \Psi_{n}^{(o)}(x)$; its evolution is governed by Eqs. (36) and (37):

$$
\begin{aligned}
\Psi_{E}^{(r)}(x, t)= & 2 \alpha \beta e^{-i \widetilde{E}_{n} t / \hbar}\left\{\Psi_{n}(x) \cos \Omega_{n} t\right. \\
& \left.+i \Psi_{n}(-x) \sin \Omega_{n}\right\} t,
\end{aligned}
$$

where $\Omega_{n}=\Delta E_{n} / \hbar$. It is seen that the electron population oscillates between both wells with a period $T_{n}=2 \pi / \Omega_{n}$, which means that the electron is forced through the central potential barrier in a time $T_{n} / 2$. Clearly, $T_{n} / 2$ is a time scale that characterizes quantum tunneling. Let us now show that $T_{n}$ is indeed intimately related to the minimum tunneling time $\tau_{\min }(E)$ for the double-well potential structure.

\section{B. Connection to $\tau_{\min }(E)$}

Let us first represent the oscillation period $T_{n}$ in terms of eigenfunctions (34) and (35) of the potential $U(x)$. A simple calculation starting from Eqs. (36) and (37) shows that [20]

$$
\int_{0}^{\infty} d x \Psi_{n}^{(e)}(x) \Psi_{n}^{(o)}(x)=-\frac{\hbar^{2}}{2 m} \frac{\mathcal{W}\left[\Psi_{n}^{(o)}(x), \Psi_{n}^{(e)}(x)\right]_{0}^{\infty}}{E_{n}^{(o)}-E_{n}^{(e)}} .
$$

The wave functions $\Psi_{n}^{(e, o)}(x)$ are bound states, so their Wronskian determinant vanishes for $x \rightarrow \infty$; at the lower limit $x=0$, we chose the normalization of these functions in a way as to guarantee a unit Wronskian. Since $E_{n}^{(o)}-E_{n}^{(e)}$ $=2 \pi \hbar / T_{n}$, we obtain from Eq. (39):

$$
T_{n}=\frac{4 \pi m}{\hbar} \int_{0}^{\infty} d x \Psi_{n}^{(e)}(x) \Psi_{n}^{(o)}(x) \text {. }
$$

This expression is still exact. However, the functions in the integrand implicitly depend (via their eigenenergies) on $T_{n}$. To get rid of this dependence, we note that their true eigenenergies deviate from their unperturbed value $E_{n}$ only by an exponentially small amount, so it appears appropriate to replace the bound states in Eq. (40) by a pair of eigen- 
functions $c(x)$ and $s(x)$ to $U(x)$ with energy $E_{n}$ that are normalized in the same way, i.e., $c(0)=s(0)^{\prime}=1$ and $c(0)^{\prime}=s(0)=0$. Then, they show a unit Wronskian determinant, $\mathcal{W}[s, c]=1[\mathrm{Eq} .(17)]$, and we obtain approximately

$$
T_{n} \approx \frac{4 \pi m}{\hbar} \int_{0}^{d} d x c(x) s(x) .
$$

Note that this operation enforces the introduction of a cutoff $d$ - unlike their counterparts $\Psi_{n}^{(e, o)}(x)$, the functions $c(x)$ and $s(x)$ are not genuinely bound states of the potential and thus diverge exponentially for $x \rightarrow \infty$. [In the example of Sec. VII C, we found it practical to choose $d$ in a way as to minimize the wave function $c(x)^{2}$ at $x=d$.]

In form (41), the period $T_{n}$ may be expressed by the minimum tunneling time $\tau_{\min }\left(E_{n}\right)$. We first remark that $\tau_{\min }\left(E_{n}\right)$ in the symmetric interval $-d<x<d$ is given by Eq. (20):

$$
\tau_{\min }\left(-d, d ; E_{n}\right)=\frac{4 m}{\hbar} \sqrt{\int_{0}^{d} d x c(x)^{2} \int_{0}^{d} d x s(x)^{2}} .
$$

[Here, we exploited the parity properties of $c(x)$ and $s(x)$.] In connection with Eq. (18), this allows us to express Eq. (41) in terms of the time scales $\tau_{\min }\left(0, d ; E_{n}\right)$ and $\tau_{\min }$ $\left(-d, d ; E_{n}\right)$ :

$$
T_{n} \approx \pi \sqrt{\tau_{\min }\left(-d, d ; E_{n}\right)^{2}-4 \tau_{\min }\left(0, d ; E_{n}\right)^{2}} .
$$

Finally, we note that $\tau_{\min }\left(0, d ; E_{n}\right)$ for opaque tunneling barriers is exponentially small compared to the minimum tunneling time for the entire structure $\tau_{\min }\left(-d, d ; E_{n}\right)$ [Eq. (32)] and thus may be omitted. This yields the estimate

$$
T_{n} \approx \pi \tau_{\min }\left(-d, d ; E_{n}\right)
$$

For tunneling barriers, the beat period $T_{n}$ in the double well is strictly coupled to $\tau_{\min }\left(E_{n}\right)$. Alternatively, we find for the level splitting the expression $\Delta E_{n} \approx 2 \hbar / \tau_{\min }\left(-d, d ; E_{n}\right)$. [Again, we emphasize that there are proposals for the tunneling time, which may lead to results much smaller in value than $\tau_{\min }\left(-d, d ; E_{n}\right)$.]

\section{EXAMPLES}

Let us now turn our attention to a few model potentials $U(x)$ in order to illustrate the theory of the minimum tunneling time $\tau_{\min }(E)$. We present some-occasionally surprising - results regarding quantum motion through simple square barriers and double-spike structures. Furthermore, we assess the quality of the minimum tunneling-time estimate for the level splitting in double well structures (Sec. VI) by numerical calculations for a parabolic double-well potential.

\section{A. Square barrier}

We begin with a study of the minimum tunneling time $\tau_{\min }(E)$ for the most popular, yet to some extent pathological barrier potential, the symmetric rectangular barrier:

$$
U(x)=U \Theta(x-b / 2) \Theta(b / 2-x) \text {. }
$$

Let us introduce the wave number $\kappa$ in the barrier,

$$
\kappa=\frac{1}{\hbar} \sqrt{2 m|E-U|},
$$

and use a pair of fundamental solutions $c(x)$ and $s(x)$ of definite parity in the barrier region $|x|<b / 2$, which is normalized in the sense of Eq. (17):

$$
c(x)=\cos (\kappa x), \quad s(x)=\frac{1}{\kappa} \sin (\kappa x) .
$$

[Here, we assume $E>U$; for $E<U$, the trigonometric functions in Eq. (47) should be replaced by the corresponding hyperbolic ones.] Then, we obtain from Eq. (20) for $\tau_{\min }(E)$ :

$$
\tau_{\min }(E)= \begin{cases}\frac{m}{\hbar \kappa^{2}} \sqrt{\sinh ^{2} \kappa b-(\kappa b)^{2}} & (E<U) \\ \frac{1}{\sqrt{3}} \frac{m b^{2}}{\hbar} & (E=U) \\ \frac{m}{\hbar \kappa^{2}} \sqrt{(\kappa b)^{2}-\sin ^{2} \kappa b} & (E>U) .\end{cases}
$$

In contrast, the corresponding dwell time $\tau_{\mathrm{S}}(E)$ [Eq. (8)] [which for this symmetric barrier coincides with the Larmor phase time $\left.\tau_{T}^{y}(E)\right]$ is only defined for $E \geqslant 0$ and reads, using the abbreviations $k^{2}=2 m E / \hbar^{2}$ and $k_{0}^{2}=2 m U / \hbar^{2}$ [8],

$$
\tau_{\mathrm{S}}(E)= \begin{cases}\frac{m k}{\hbar \kappa} \frac{2 \kappa b\left(\kappa^{2}-k^{2}\right)+k_{0}^{2} \sinh 2 \kappa b}{4 \kappa^{2} k^{2}+k_{0}^{4} \sinh ^{2} \kappa b} & (0<E<U) \\ \frac{m b}{\hbar k_{0}} \frac{1+k_{0}^{2} b^{2} / 3}{1+k_{0}^{2} b^{2} / 4} & (E=U) \\ \frac{m k}{\hbar \kappa} \frac{2 \kappa b\left(\kappa^{2}+k^{2}\right)-k_{0}^{2} \sin 2 \kappa b}{4 \kappa^{2} k^{2}+k_{0}^{4} \sin ^{2} \kappa b} & (E>U) .\end{cases}
$$

Figure 3 shows that the minimum tunneling time $\tau_{\min }(E)$ for the square barrier smoothly rises with decreasing energy $E$. As expected, in the classical limit $E \gg U, \tau_{\min }(E)$ merges into the classical time of flight $\tau_{\mathrm{cl}}(E)=b / \mathrm{v}_{\mathrm{cl}}(E)$, whereas in the limit of particle tunneling $(E \ll U)$, the minimum tunneling time grows exponentially with both $\kappa$ and $b$, thus confirming the asymptotic formulas put forward in Sec. V. For $E$ $=U, \tau_{\min }(E)$ passes smoothly through its universal value for narrow barriers (24). We note that the usual dwell time $\tau_{\mathrm{S}}(E)[\mathrm{Eq} .(49)]$ in the tunneling regime is characterized by a radically different behavior.

\section{B. Double delta spike}

We now switch to a further example where the minimum tunneling time $\tau_{\min }(E)$ shows a rather peculiar behavior. The model potential barrier we have in mind consists merely of two delta "spikes" forming a symmetric quantum well:

$$
U(x)=U[\delta(x-b / 2)+\delta(x+b / 2)] .
$$

Resonant tunneling through the potential spikes causes a conspicuous pattern in the transmission amplitude $T(E)$, and 


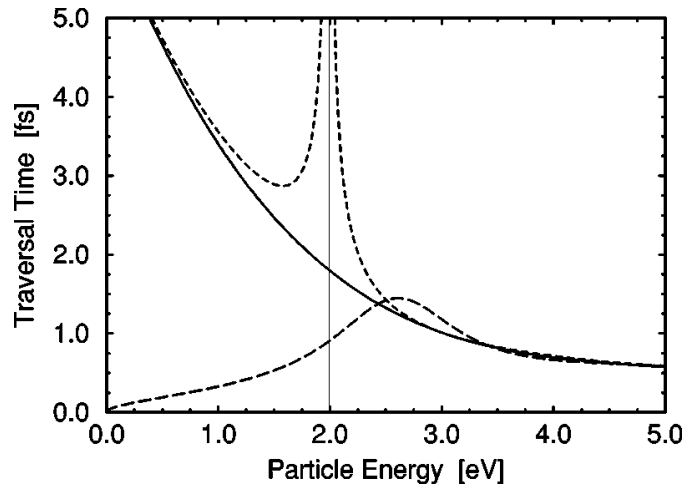

FIG. 3. Minimum tunneling time $\tau_{\min }(E)$ [Eq. (48)] for a rectangular barrier of width $b=6 \AA$ and height $U=2 \mathrm{eV}$ as a function of the particle energy $E$ (solid line). For comparison, the classical time of flight $\tau_{\mathrm{cl}}(E)(E>U)$, and the WKB approximation $\tau_{\min }^{\mathrm{WKB}}(E)$ [Eq. (32)] for barrier penetration $(E<U)$ are additionally plotted (short lines). The entirely different behavior of the corresponding dwell time $\tau_{\mathrm{S}}(E)=\tau_{T}^{y}(E)$ [Eq. (49)] in the tunneling regime is evident (long dashed line).

we should thus expect that these resonances reveal themselves also in the tunneling-time spectrum. Indeed, this property holds for Smith's dwell time $\tau_{\mathrm{S}}(E)$ [Eq. (8)], which here equals the Larmor time scale $\tau_{T}^{y}(E)$ [Eq. (14)], and for this particular barrier is given by

$\tau_{\mathrm{S}}(E)=\frac{2 m}{\hbar} \frac{k b\left(\kappa^{2}+2 k^{2}\right)+\kappa \sin k b(2 k \sin k b-\kappa \cos k b)}{4 k^{4}+\kappa^{2}(2 k \cos k b+\kappa \sin k b)^{2}}$

Here, $k^{2}=2 m E / \hbar^{2}$, and $\kappa=2 m U / \hbar^{2}$ is a measure for the strength of the spike potentials.

From Sec. IV we infer that the minimum tunneling time $\tau_{\min }(E)$ for the well solely depends on the wave functions $\Psi_{E}(x)$ in the interior of the barrier $|x|<b / 2$, which are completely unaffected by the presence of the delta spikes! Therefore, $\tau_{\min }(E)$ is independent of the spike strength $U$ and hence given by its free-particle value (48):

$$
\tau_{\min }(E)=\frac{m}{\hbar k^{2}} \sqrt{(k b)^{2}-\sin ^{2} k b} .
$$

In Fig. 4, we display both tunneling time scales as a function of $E$. As anticipated, $\tau_{\min }(E)$ depends on the internal structure of the potential $U(x)$ rather than its transmission properties $T(E)$, which affect Smith's dwell time $\tau_{\mathrm{S}}(E)$ in a considerable manner (Sec. II B). [The Larmor time scales are entirely based on the transmission and reflection amplitudes $T(E)$ and $R(E)$ of $U(x)$, see Eqs. (10) and (11).]

\section{Parabolic double well}

Finally, we would like to verify the connection between the minimum tunneling time $\tau_{\min }\left(E_{n}\right)$ and the level splitting $\Delta E_{n}$ in a symmetric double-well potential $U(x)=U(-x)$ that we established in Sec. VI. For this purpose, we construct

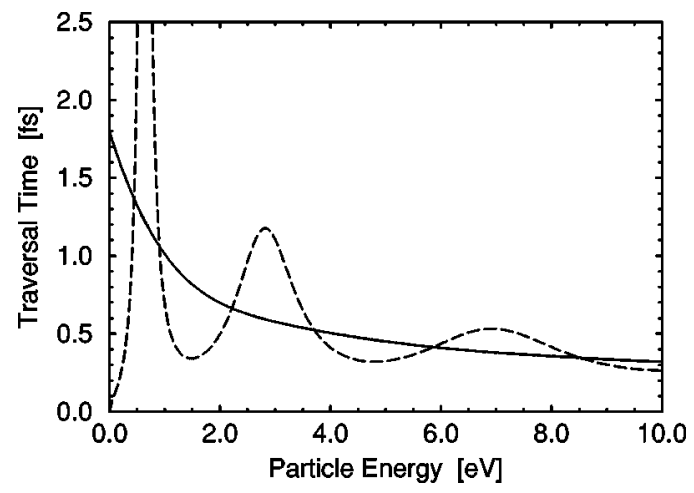

FIG. 4. Tunneling times for motion through a symmetric potential well demarcated by two identical delta spikes $U \delta(x \pm b / 2)$ as a function of energy $E$. Solid line: $\tau_{\min }(E)$ [Eq. (52)]. Dashed line: $\tau_{\mathrm{S}}(E)=\tau_{T}^{y}(E)$ [Eq. (51)]. Note that $\tau_{\min }(E)$ may exceed the dwell time. (Parameters used: $U=4 \mathrm{eV} \AA, b=6 \AA$ ).

such a double well in a comparatively simple manner by matching parts of parabolic potentials:

$$
U(x)= \begin{cases}\frac{m \omega^{2}}{2}(x+b)^{2} & (x<-c) \\ U_{0}-\frac{m \omega^{2}}{2} x^{2} & (|x|<c) \\ \frac{m \omega^{2}}{2}(x-b)^{2} & (x>c) .\end{cases}
$$

For a continuously differentiable potential function $U(x)$, we have to set $c=b / 2$ and $U_{0}=m \omega^{2} b^{2} / 4$ in Eq. (53). The shape of this parabolic double well is depicted in Fig. 2.

Here, we are interested in a calculation of the exact level splitting $\Delta E_{0}$ and corresponding minimum tunneling-time estimate (44) for the lowest-lying doublet, which emerges from the unperturbed oscillator well ground state at $E_{0}$ $=\hbar \omega / 2$. Clearly, the height $U_{0}$ and the extension of the central tunneling barrier may be tuned by changing the well separation $2 b$. As a useful first approximation to $\Delta E_{0}$, we employ a semiclassical formula taken from Landau's textbook [21]:

$$
\Delta E_{0}=\frac{\hbar \omega}{\pi} \exp \left\{-\frac{1}{\hbar}\left|S_{\mathrm{cl}}\left(-a, a ; E_{0}\right)\right|\right\} .
$$

Here, $\pm a$ denote the turning points of classical motion, and $S_{\mathrm{cl}}\left(-a, a ; E_{0}\right)$ represents the classical action for barrier penetration (23). [Note that our less sophisticated estimate (32) is not directly applicable here as it has not been corrected for the effects of turning points. However, both expressions agree in their exponential dependence.]

Despite its simple structure, the eigenstates $\Psi_{n}^{(e, o)}(x)$ [Eqs. (34) and (35)] of the double-well potential $U(x)$ [Eq. (53)] are available only through numerical computation. They may be represented in terms of parabolic cylinder functions [18] properly matched at $x= \pm c$. In a recursive procedure, we determined the exact eigenenergies $E_{0}^{(e)}$ and $E_{0}^{(o)}$ of the lowest levels of $U(x)$ and compiled a list of the corresponding level splittings $\Delta E_{0}$ for several values of the dimensionless separation parameter $\beta=\sqrt{2 m \omega / \hbar} b$. Numerical values for the minimum tunneling time $\tau_{\min }\left(-d, d ; E_{0}\right)$ [Eq. 
TABLE I. Numerical results for the level splitting in a symmetric parabolic double well [Eq. (53)]. The first column specifies a dimensionless measure $\beta=\sqrt{2 m \omega / \hbar} b$ for the separation of both wells; next, the corresponding crest $U_{0}$ of the central potential barrier is given in terms of $\hbar \omega$. The following three columns display the level splitting $\Delta E_{0}$ for the oscillator ground state $(n=0)$ in units of $\hbar \omega$. The exact value is presented in the left column, whereas the approximation to $\Delta E_{0}$ gained via the tunneling-time scheme is stated in the central column. Semiclassical estimate (54) for $\Delta E_{0}$ is shown in the right column. To the extreme right, the relative error of the minimum tunneling time approach is displayed.

\begin{tabular}{cccccc}
\hline \hline$\beta$ & $U_{0}$ & $\Delta E_{0}($ exact $)$ & $\Delta E_{0}\left[\operatorname{via} \tau_{\min }(E)\right]$ & $\Delta E_{0}$ (semicl.) & Relative error \\
\hline 3 & 1.125 & $4.86088 \times 10^{-2}$ & $5.32624 \times 10^{-2}$ & $4.46249 \times 10^{-2}$ & $9.57 \times 10^{-2}$ \\
4 & 2.000 & $2.91743 \times 10^{-3}$ & $2.98873 \times 10^{-3}$ & $2.58577 \times 10^{-3}$ & $2.44 \times 10^{-2}$ \\
5 & 3.125 & $6.46587 \times 10^{-5}$ & $6.49829 \times 10^{-5}$ & $5.80656 \times 10^{-5}$ & $5.01 \times 10^{-3}$ \\
6 & 4.500 & $5.65062 \times 10^{-7}$ & $5.65553 \times 10^{-7}$ & $5.13431 \times 10^{-7}$ & $8.69 \times 10^{-4}$ \\
7 & 6.125 & $1.97591 \times 10^{-9}$ & $1.97615 \times 10^{-9}$ & $1.80841 \times 10^{-9}$ & $1.23 \times 10^{-4}$ \\
8 & 8.000 & $2.78036 \times 10^{-12}$ & $2.78040 \times 10^{-12}$ & $2.55579 \times 10^{-12}$ & $1.41 \times 10^{-5}$ \\
\hline \hline
\end{tabular}

(42)] were established in a second series of calculations. To this end, carrying out the program of Sec. VI B we first determined properly normalized pairs of eigenfunctions $c(x)$ and $s(x)$ to $U(x)$ with energy $E_{0}=\hbar \omega / 2$. Next, we selected cutoffs $d$ for the integration in Eq. (41): Our simple criterion identified $d$ with the minimum of the absolute value of the approximate solution $c(x)$ in the exterior sector $x>b$, i.e., we chose the largest value of $d$ so that $c(d) c(d)^{\prime}=0$. [These cutoffs are usually located far in the outer barriers of the double well, thus indicating good performance of approximation (41), see Fig. 2.] The minimum tunneling time then was computed by integration (42) and subsequently converted into an estimate for $\Delta E_{0}$ by means of Eq. (44).

Table I gathers results of these calculations for parabolic double wells of different separation and barrier penetrability. For comparison, the exact level splittings $\Delta E_{0}$ are complemented with corresponding minimum tunneling-time estimates (44) and the less accurate semiclassical formula (54). We infer that except for very shallow barriers (small values of $\beta$ ), the tunneling-time estimate indeed presents a splendid approximation to the true level splitting (extreme right column in Table I), thus confirming the significance of $\tau_{\min }(E)$ as a measure for the duration of quantum motion in these structures.

\section{CONCLUSION}

Pursuing a variational approach to the long-standing tunneling-time problem, in this paper we presented the concept of the minimum tunneling time $\tau_{\min }(E)$, a time scale of quantum motion that holds for stationary one-dimensional problems. It is defined as the minimum amount of time required by the probability current in order to replace the particle number present in a given interval of space $a<x<b$. Therefore, $\tau_{\min }(E)$ bears a close relationship to the idea of the dwell time originally proposed by Smith, which essentially represents an analogous quantity for outgoing waves. Both approaches are rooted in the hydrodynamical interpretation of quantum mechanics advocated in particular by Bohm.

As a major feature of the minimum tunneling-time ansatz, we emphasized that the quantity $\tau_{\min }(E)$ represents a genuinely local time scale of motion that, apart from the particle energy $E$, solely depends on the topography of the potential
$U(x)$ in the sector of interest $a<x<b$, whereas most contenders for the rank of the tunneling time scale, including the dwell time and the ubiquitous set of Larmor time scales, implicitly rely on boundary conditions imposed onto the underlying wave functions. (Nevertheless, the minimum tunneling time may be expressed as a functional of the set of Larmor clock readings.) The variational principle leads to simple and elegant formulas for $\tau_{\min }(E)$. Asymptotic expressions for the minimum tunneling time are available in the semiclassical limit: In the case of classically allowed motion, $\tau_{\min }(E)$ gradually merges into the classical time of flight, and thus matches a basic demand for a valid tunneling time scale. For the opposite case of barrier penetration, the minimum tunneling time grows exponentially with the classical action for barrier traversal (32). Qualitatively speaking, $\tau_{\min }(E)$ is much shorter than the lifetime of a corresponding metastable state: Whereas $\tau_{\min }(E)$ is inversely proportional to the modulus $|T(E)|$ of the semiclassical transmission amplitude, the lifetime of the metastable state grows with $|T(E)|^{-2}$. At the same time, $\tau_{\min }(E)$ generally lasts much longer than the semiclassical instanton or bounce tunneling time, which is defined as the classical traversal time for a particle of energy $-E$ moving in the inverted potential barrier $-U(x)$. The results of Sec. VI suggest that the variationally defined tunneling time $\tau_{\min }(E)$ corresponds to a periodical process where a particle tunnels through a barrier in a coherent fashion, whereas the decay of a metastable state proceeds in an incoherent, irreversible manner.

Apart from the formal elegance of the variational method, the minimum tunneling-time approach has the advantage of being related to experimentally accessible quantities. As a physical process that allows for the determination of $\tau_{\min }(E)$, we identified the energy level splitting in a symmetric double-well potential caused by tunneling of particles through the central potential barrier separating both quantum wells. These level splittings, and thus the minimum tunneling time for the double-well structure, are available from spectroscopic measurements. We finally remark that a quite similar situation is realized in nature by electron exchange during the scattering of protons on neutral hydrogen atoms [22].

\section{ACKNOWLEDGMENT}

One of us (C.B.) has benefited from the support of the "Studienstiftung des Deutschen Volkes.' 
[1] E.H. Hauge and J.A. Støvneng, Rev. Mod. Phys. 61, 917 (1989).

[2] R. Landauer and Th. Martin, Rev. Mod. Phys. 66, 217 (1994).

[3] Tunneling and its Implications, Proceedings of the Adriatico Research Conference, Trieste, 1996, edited by D. Mugnai, A. Ranfagni, and L. S. Schulman (World Scientific, Singapore, 1997).

[4] G. Nimtz and A. Enders, J. Phys. I 2, 1693 (1992).

[5] Proceedings of the Workshop on Superluminal Velocities, Cologne, 1998, edited by P. Mittelstaedt and G. Nimitz [Ann. Phys. (Leipzig) 7, 585 (1998)].

[6] A.M. Steinberg, P.G. Kwiat, and R.Y. Chiao, Phys. Rev. Lett. 71, 708 (1993).

[7] E.P. Wigner, Phys. Rev. 98, 145 (1955).

[8] M. Büttiker, Phys. Rev. B 27, 6178 (1983).

[9] M. Büttiker, in Electronic Properties of Multilayer and LowDimensional Semiconductor Structures, edited by J.M. Chamberlain (Plenum, New York, 1990), p. 297.

[10] C. Bracher, J. Phys. B 30, 2717 (1997).

[11] One might decline this point of view, and instead advocate the notion that the particle moves inside a single well, where it is kept for an extended time due to reflection at the central barrier [see, for example, R. Landauer, Nature (London) 341, 567 (1989)]. What we want to point out here is the coherent nature of the resonant tunneling process that manifests itself, e.g., in the duration of oscillation period (54) that is linked to the modulus of the transmission amplitude $|T(E)|$ rather than the transmission probability $|T(E)|^{2}$, as might be naively expected.
[12] F.T. Smith, Phys. Rev. 118, 349 (1960).

[13] C.R. Leavens and G.C. Aers, in Scanning Tunneling Microscopy III, edited by R. Wiesendanger and H.J. Güntherodt (Springer, Berlin, 1993), p. 105.

[14] A.I. Baz', Yad. Fiz. 4, 252 (1966) [Sov. J. Nucl. Phys. 4, 182 (1967)]; 5, 229 (1967) [5, 161 (1967)].

[15] M. Deutsch and J.E. Golub, Phys. Rev. A 53, 434 (1996).

[16] Ph. Balcou and L. Dutriaux, Phys. Rev. Lett. 78, 851 (1997).

[17] One may raise the objection that all available information concerning a physical system is contained in its quantum wave function. Although this statement is obviously correct, it is not an easy task to tailor the wave function to the needs of various tunneling-time proposals, including the dwell and Larmor times (Sec. II), which implicitly employ outgoing-wave solutions. Furthermore, there exist quantum processes whose evolution is governed by characteristic time scales, which are independent of the actual choice of the wave function. Resonant tunneling in symmetric double-well barrier structures (Sec. VI) presents a notable example.

[18] Handbook of Mathematical Functions, edited by M. Abramowitz and I.A. Stegun (Dover, New York, 1972).

[19] R.P. Feynman, R.B. Leighton, and M. Sands, The Feynman Lectures on Physics (Addison-Wesley, Reading, 1965), Vol. 3, Chap. 9.

[20] A. Messiah, Quantum Mechanics (North-Holland, Amsterdam, 1964), Vol. I, p. 99.

[21] L.D. Landau and E.M. Lifshitz, Quantum Mechanics (Pergamon, London, 1958), p. 177.

[22] G.J. Lockwood and E. Everhart, Phys. Rev. 125, 567 (1962). 